\title{
Age, participation in competitive sports, bony lesions, ALPSA lesions, $>1$ preoperative dislocations, surgical delay and ISIS score $>3$ are risk factors for recurrence following arthroscopic Bankart repair: a systematic review and meta-analysis of 4584 shoulders
}

\author{
Lukas P. E. Verweij ${ }^{1,2,3}$. Sanne H. van Spanning ${ }^{4}$ Adriano Grillo ${ }^{4}$. Gino M. M. J. Kerkhoffs ${ }^{1,2,3}$. \\ Simone Priester-Vink ${ }^{5}$. Derek F. P. van Deurzen ${ }^{4}$. Michel P. J. van den Bekerom ${ }^{4,6}$
}

Received: 11 June 2021 / Accepted: 13 August 2021 / Published online: 22 August 2021

(c) The Author(s) 2021

\begin{abstract}
Purpose Determining the risk of recurrent instability following an arthroscopic Bankart repair can be challenging, as numerous risk factors have been identified that might predispose recurrent instability. However, an overview with quantitative analysis of all available risk factors is lacking. Therefore, the aim of this systematic review is to identify risk factors that are associated with recurrence following an arthroscopic Bankart repair.

Methods Relevant studies were identified by searching PubMed, Embase/Ovid, Cochrane Database of Systematic Reviews/ Wiley, Cochrane Central Register of Controlled Trials/Wiley, CINAHL/Ebsco, and Web of Science/Clarivate Analytics from inception up to November 12th 2020. Studies evaluating risk factors for recurrence following an arthroscopic Bankart repair with a minimal follow-up of 2 years were included.

Results Twenty-nine studies met the inclusion criteria and comprised a total of 4582 shoulders ( 4578 patients). Meta-analyses were feasible for 22 risk factors and demonstrated that age $\leq 20$ years $(\mathrm{RR}=2.02 ; P<0.00001)$, age $\leq 30$ years $(\mathrm{RR}=2.62$; $P=0.005)$, participation in competitive sports ( $\mathrm{RR}=2.40 ; P=0.02$ ), Hill-Sachs lesion ( $\mathrm{RR}=1.77 ; P=0.0005)$, off-track Hill-Sachs lesion $(\mathrm{RR}=3.24 ; P=0.002)$, glenoid bone loss $(\mathrm{RR}=2.38 ; P=0.0001)$, ALPSA lesion $(\mathrm{RR}=1.90 ; P=0.03),>1$ preoperative dislocations $(\mathrm{RR}=2.02 ; P=0.03),>6$ months surgical delay $(\mathrm{RR}=2.86 ; P<0.0001)$, $\mathrm{ISIS}>3(\mathrm{RR}=3.28$; $P=0.0007)$ and ISIS $>6(\mathrm{RR}=4.88 ; P<0.00001)$ were risk factors for recurrence. Male gender, an affected dominant arm, hyperlaxity, participation in contact and/or overhead sports, glenoid fracture, SLAP lesion with/without repair, rotator cuff tear, $>5$ preoperative dislocations and using $\leq 2$ anchors could not be confirmed as risk factors. In addition, no difference was observed between the age groups $\leq 20$ and $21-30$ years.

Conclusion Meta-analyses demonstrated that age $\leq 20$ years, age $\leq 30$ years, participation in competitive sports, Hill-Sachs lesion, off-track Hill-Sachs lesion, glenoid bone loss, ALPSA lesion, $>1$ preoperative dislocations, $>6$ months surgical delay from first-time dislocation to surgery, ISIS $>3$ and ISIS $>6$ were risk factors for recurrence following an arthroscopic Bankart repair. These factors can assist clinicians in giving a proper advice regarding treatment.

Level of evidence Level IV.
\end{abstract}

Keywords Shoulder $\cdot$ Anterior dislocation $\cdot$ Instability $\cdot$ Risk $\cdot$ Bankart

\begin{abstract}
Abbreviations
ISIS Instability severity index score

MINORS Methodological index for non-randomized studies
\end{abstract}

Lukas P. E. Verweij

1.p.verweij@amsterdamumc.nl

Extended author information available on the last page of the article
ALPSA Anterior labroligamentous periosteal sleeve avulsion

GLAD Glenolabral articular disruption

SLAP Superior labral anterior posterior

MSTS Multiple subscapularis tendon sign

RR Risk ratios

CI Confidence interval 


\section{Introduction}

Shoulder instability is characterized by dislocation or subluxation of the glenohumeral joint or a feeling of apprehension. The estimated incidence rate in the United States is 23.9 per 100,000 person-years and the cause is often traumatic [59]. Furthermore, over $95 \%$ of shoulder dislocations occur in the anterior direction, in contrast to the less frequently occurring posterior and inferior dislocations $[50,59]$. Shoulder dislocations limit patients in activities of daily living and sports and are associated with development of osteoarthritis $[51,60]$. In addition, recurrent instability includes high social costs and performing operative treatment following a first-time dislocations is demonstrated to be cost-effective $[9,47]$. In a prospective study with 25 years of follow-up, Hovelius et al. demonstrated a recurrence rate of up to $60 \%$ following nonoperative treatment, which generally consists of scapula and rotator cuff training $[8,19]$. Operative treatment may have been beneficial for these patients.

The most commonly performed surgical treatment options include repair of the labrum with/without tenomyodesis of the infraspinatus tendon (remplissage) and bone augmentation of the glenoid [50]. Counseling patients for operative treatment can be challenging as the risks and benefits for each individual patient must be weighed. The arthroscopic labral repair demonstrates a recurrence rate of $16 \%$, whereas the open bone augmentation procedures are more effective and show a recurrence rate of $2-6 \%[21,55]$. However, these procedures are more invasive and demonstrate a complication rate of $5-14 \%$ compared to $<2 \%$ following the arthroscopic repair [21, 55]. Numerous studies have investigated if glenoid bone loss has a cut-off value that can advise professionals for which cases to perform a bone augmentation procedure $[42,52]$. However, an objective cut-off value that predicts recurrent instability has yet to be found and the current methods only measure bone loss in $2 \mathrm{D}$, therefore not taking the 3D morphology into account [52]. Furthermore, other methods that might be able to determine recurrence risk are proposed. These include the instability severity index score (ISIS), glenoid morphology (i.e. concavity, version, inclination), an off-track Hill-Sachs lesion and translation of the humeral head $[2,11,27,29,56]$. These methods, or a combination of these methods, seem promising to objectively determine recurrence risk in the future. Currently, mainly risk factors based on group averages are used to predict recurrence risk following an arthroscopic Bankart repair. The most recent systematic review evaluating these factors was published almost a decade ago by Randelli et al. [38]. This review demonstrates that there is no consensus regarding which risk factors predispose recurrence and therefore demands a quantitative analysis. In addition, since then many studies evaluating new risk factors have been published that need to be included in the overview $[10,24,30]$. Therefore, the aim of this systematic review is to identify risk factors that are associated with recurrence following an arthroscopic Bankart repair.

\section{Materials and methods}

This systematic review was carried out in accordance with the PRISMA (Preferred Reporting Items for Systematic Reviews and Meta-Analyses) protocol and registered with the PROSPERO database (registration number: CRD42020212423)[28].

\section{Literature search}

Relevant studies were identified by searching PubMed, Embase/Ovid, Cochrane Database of Systematic Reviews/ Wiley, Cochrane Central Register of Controlled Trials/ Wiley, CINAHL/Ebsco, and Web of Science/Clarivate Analytics from inception up to November 12th 2020. The following terms, including synonyms and closely related words, were used as index terms or free-text words: 'shoulder', 'dislocation', 'Bankart' and 'recurrence' or 'tear'. Full search strategies for all databases are available in supplementary 1. No language or other restrictions were applied to any of the searches. Duplicate articles were excluded by the information specialist using EndNote X8 (Clarivate analytics, Philadelphia, Pennsylvania, United States). Studies that met the inclusion criteria were screened full-text. In addition, the reference list of each study was assessed to find other possibly relevant studies. Both title/abstract screening and full-text screening were performed by two authors (L.P.E. V. and S.H. S.), with the use of Rayyan [32]. Any disagreement was resolved by discussion and consensus. If the authors were unable to reach a consensus, a third author (M.P.J. B.) would give final judgment.

\section{Inclusion and exclusion criteria}

Prospective and retrospective cohort studies evaluating risk factors for recurrence following an arthroscopic Bankart repair with a minimum follow-up of 2 years were included. Recurrence was defined as a complete anterior shoulder dislocation or subluxation. Comparative studies were only included when independent risk factors were identified. Only articles written in the English, Dutch, German or Italian language were included. Studies were excluded if the mean age was less than 18 years or when the main focus was patients with posterior, multidirectional or atraumatic instability. In addition, reviews, cadaveric studies, software simulations, 
case reports, animal studies, abstracts, book chapters and studies evaluating recurrence following other treatments than an arthroscopic Bankart repair were excluded. When the same cohort of patients was used, the study with the longest follow-up was included.

\section{Quality appraisal}

The methodological quality of the selected studies was assessed using the Methodological Index for Non-Randomized Studies (MINORS) tool [44]. The included comparative studies that determined independent risk factors were seen as non-comparative studies during the assessment. A non-randomized non-comparative study can earn a maximum of 16 points using the MINORS tool. The assessment was performed by two authors (L.P.E. V. and S.H. S.). Following the assessment, the authors compared the results to create a final rating for the individual studies. If the authors were unable to reach a consensus, a third author (M.P.J. B.) would give final judgment.

\section{Data extraction}

Extracted baseline patient characteristics included sample size, gender, mean age at surgery and follow-up. The primary outcome was recurrence following an arthroscopic Bankart repair. An arthroscopic Bankart repair was defined as any form of arthroscopic anterior capsulolabral repair without tenomyodesis of the infraspinatus tendon. Data of risk factors were extracted if the proportion of recurrence for patients with and without a specific risk factor could be extracted or calculated. If proportions could not be extracted or calculated for any risk factor in studies published after 2010, the authors were sent an email and asked to share the data. Risk factors included age at surgery, gender, if the dominant arm was affected, hyperlaxity as defined by the authors, participation in contact and/or overhead sports, participation in competitive sports, Hill-Sachs lesions, if the Hill-Sachs lesion was off-track, glenoid bone loss, glenoid fractures, Anterior Labroligamentous Periosteal Sleeve Avulsion (ALPSA) lesions, Glenolabral Articular Disruption (GLAD) lesions, Superior Labral Anterior Posterior (SLAP) lesions, rotator cuff tears, time from first-time dislocation to surgical treatment, number of preoperative dislocations, Multiple Subscapularis Tendon Sign (MSTS) and ISIS. As variety in definition for specific risk factors was present, the definition of the original articles was adopted. Arthroscopy was considered the gold standard to identify any lesion. However, lesions identified with either radiographs, CT or MRI were extracted as well. If percentage of glenoid bone loss was measured, it was considered to be present when $>5 \%$ was measured with any glenoid bone loss measuring method using CT, MRI or during arthroscopy [10, 52]. Data were extracted to Excel (Microsoft Corporation. Microsoft Excel [Internet]. 2016. Available from: https://office.microsoft.com/excel).

\section{Statistical analysis}

Patient characteristics and length of follow-up were pooled by calculation of weighted means and pooled standard deviations. If the standard deviation was not reported, it was estimated with the range and the sample size according to Walter et al. [54]. Furthermore, if the mean was not reported, it was estimated using the median, range and sample size according to Hozo et al. [20]. Proportion of recurrence was calculated for patient groups with and without a specific risk factor. If only an odds ratio was reported, the proportions were calculated when sufficient variables were available for the calculation. If possible, risk factor data were pooled to perform meta-analyses, including $\geq 2$ studies. Since small differences were observed for the risk factor age at time of surgery (i.e. age $<20$ and age $\leq 20$ years), a deviation of one year was accepted to pool the data. Proportions were compared by use of $\mathrm{X}^{2}$ tests. Review Manager version 5.3 (the Nordic Cochrane Center, Copenhagen, Denmark) was used to perform meta-analyses and calculate risk ratios (RR) with 95\% confidence interval (CI). Heterogeneity between studies was assessed by use of the $I^{2}$ statistic[18].

\section{Results}

\section{Screening and study characteristics}

After duplicates were removed, the titles and abstracts of 3584 studies were screened (Fig. 1). Sixty-seven studies were included in the full-text screening of which 28 studies met the inclusion criteria. Three study groups that published their manuscript after 2010 were asked to share their data as proportions could not be calculated. One research group replied, which created a total of 29 inclusions for analysis [46]. Reasons for exclusion during the full-text screening are listed in Fig. 1. Seven prospective [4, 5, 10, 37, 46, 48, 53] and 22 retrospective studies [1, 3, 6, 7, 14-17, 22-25, 30, 31, 33, 35, 36, 43, 45, 49, 57, 58] were included (Table 1). The included studies comprised a combined sample size of 4584 shoulders (range 51-670) in 4564 patients (supplementary 2). The weighted mean age at surgery was 27.2 years (range 10-67) and $82 \%$ of patients were male. The weighted mean follow-up was 6.3 years (range 2-14.3) and during this follow-up period a weighted recurrence rate of $17 \%$ (range $6 \%-35 \%$ ) was observed. The MINORS ranged from 7 to 14 


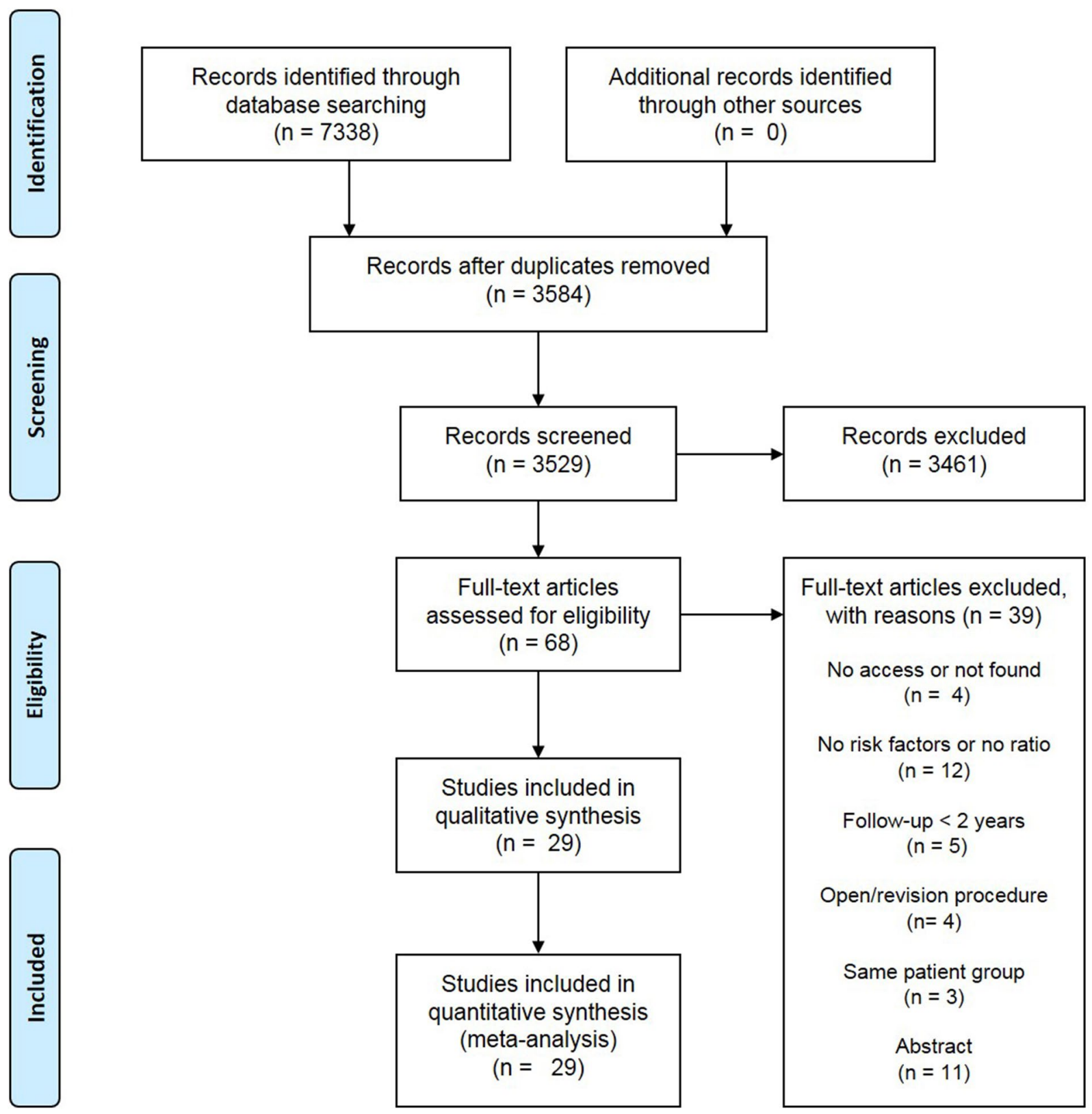

Fig. 1 Flow diagram

points (Table $1 \&$ supplementary 3 ). In total, 22 risk factors could be quantitatively analyzed.

\section{Patient factors}

Meta-analyses were feasible for patient age, gender, an affected dominant arm and hyperlaxity. Fifteen studies (2739 shoulders) demonstrated a higher recurrence risk in patients aged $\leq 20$ years compared to patients $>20$ years (RR=2.02; $P<0.00001 ; I^{2}=58 \%$; Fig. 2); five studies (588 shoulders) demonstrated a higher recurrence risk in patients aged $\leq 30$ years compared to patients $>30$ years $(R R=2.62$;
$P=0.005 ; I^{2}=57 \%$; supplementary 4, Fig. 1$)$; seven studies (622 shoulders) demonstrated no difference in recurrence risk when comparing patients aged $\leq 20$ years and 21-30 years $\left(\mathrm{RR}=1.66\right.$; n.s.; $I^{2}=65 \%$; supplementary 4 , Fig. 2); 18 studies (2973 shoulders) demonstrated no difference in recurrence risk when comparing males and females (RR=1.10; n.s.; $I^{2}=43 \%$; supplementary 4, Fig. 3); seven studies (1008 shoulders) demonstrated no difference in recurrence risk when comparing patients where the dominant arm was affected and patients where the non-dominant arm was affected (RR $=0.76$; n.s.; $I^{2}=0 \%$; supplementary 4, Fig. 4); 10 studies (1670 shoulders) demonstrated no 
Table 1 Study characteristics

\begin{tabular}{|c|c|c|c|c|c|c|c|c|}
\hline Author & Year & Design & Sample size & Male $(\%)$ & $\begin{array}{l}\text { Mean age at } \\
\text { operation (y) }\end{array}$ & Mean follow-up (y) & Recurrence $(\%)$ & MINORS \\
\hline Hayashida et al. [17] & 1998 & $\mathrm{R}$ & 82 & 77 & $21.0 \pm 7.6$ & $3.3 \pm 0.8$ & 18 & 7 \\
\hline Calvo et al. [5] & 2005 & $\mathrm{P}$ & 61 & 85 & $27.5 \pm 10.8$ & $3.7 \pm 1.4$ & 18 & 12 \\
\hline Porcellini et al. [37] & 2009 & $\mathrm{P}$ & 385 & 72 & $28.7 \pm 8.2$ & $3.0 \pm 0.0$ & 8 & 8 \\
\hline Flinkkilä et al. [14] & 2010 & $\mathrm{R}$ & 174 & 72 & $28.0 \pm 9.0$ & $4.6 \pm 1.1$ & 19 & 8 \\
\hline Voos et al. [53] & 2010 & $\mathrm{P}$ & 73 & 84 & $32.6 \pm 8.4$ & $2.8 \pm 0.4$ & 15 & 12 \\
\hline Van der Linde et al. [48] & 2011 & $\mathrm{P}$ & 68 & 66 & $31.0 \pm 7.8$ & $9.0 \pm 0.4$ & 35 & 12 \\
\hline Sommaire et al. [45] & 2012 & $\mathrm{R}$ & 77 & 79 & $27.5 \pm 8.7$ & $3.7 \pm 0.3$ & 16 & 7 \\
\hline Bessiere et al. [3] & 2013 & $\mathrm{R}$ & 51 & 86 & $26.0 \pm 6.9$ & $5.3 \pm 0.4$ & 24 & 9 \\
\hline Bouliane et al. [4] & 2014 & $\mathrm{P}$ & 100 & 77 & $25.2 \pm 9.0$ & $2.0 \pm 0.0$ & 6 & 14 \\
\hline Shibataet al. [43] & 2014 & $\mathrm{R}$ & 102 & 81 & $25.7 \pm 5.2$ & $5.6 \pm 1.6$ & 9 & 10 \\
\hline Phadnis et al. [35] & 2015 & $\mathrm{R}$ & 141 & 78 & $27.1 \pm 8.9$ & $3.9 \pm 2.2$ & 13 & 10 \\
\hline Gasparini et al. [15] & 2016 & $\mathrm{P}$ & 143 & 90 & $25.0 \pm 8.1$ & $7.5 \pm 2.3$ & 23 & 8 \\
\hline Aboalata et al. [1] & 2017 & $\mathrm{R}$ & 143 & 75 & $28.2 \pm 8.3$ & 13.3 & 18 & 7 \\
\hline Nakagawa et al. [30] & 2017 & $\mathrm{R}$ & 113 & 88 & $18.3 \pm 3.8$ & $>2$ & 20 & 8 \\
\hline Pogorzelski et al. [36] & 2018 & $\mathrm{R}$ & 62 & 84 & $21.5 \pm 3.5$ & $6.5 \pm 3.5$ & 16 & 7 \\
\hline Lee et al. [24] & 2018 & $\mathrm{R}$ & 170 & 89 & $22.7 \pm 3.0$ & $3.1 \pm 0.6$ & 19 & 11 \\
\hline Yang et al. [57] & 2018 & $\mathrm{R}$ & 160 & 90 & $27.7 \pm 6.4$ & $6.4 \pm 2.0$ & 14 & 10 \\
\hline Gul et al. [16] & 2019 & $\mathrm{R}$ & 62 & 52 & $26.7 \pm 8.0$ & $2.4 \pm 1.1$ & 8 & 10 \\
\hline Chan et al. [6] & 2019 & $\mathrm{R}$ & 131 & 91 & $26.8 \pm 5.3$ & $>2$ & 26 & 10 \\
\hline Loppini et al. [25] & 2019 & $\mathrm{R}$ & 670 & 85 & $27.0 \pm 3.5$ & $8.8 \pm 1.3$ & 17 & 8 \\
\hline Iban et al. [22] & 2019 & $\mathrm{R}$ & 142 & 83 & $35.5 \pm 7.9$ & $5.3 \pm 1.2$ & 14 & 9 \\
\hline Thomazeau et al. [46] & 2019 & $\mathrm{P}$ & 125 & 68 & $30.2 \pm 9.0$ & $9.0 \pm 0.0$ & 19 & 10 \\
\hline Vermeulen et al. [49] & 2019 & $\mathrm{R}$ & 147 & 76 & $30.0 \pm 11.1$ & $6.3 \pm 1.7$ & 22 & 8 \\
\hline Ono et al. [31] & 2019 & $\mathrm{R}$ & 51 & 88 & $27.0 \pm 7.3$ & $10.1 \pm 0.7$ & 31 & 7 \\
\hline Kanatli et al. [23] & 2019 & $\mathrm{R}$ & 87 & 86 & $28.4 \pm 10.8$ & $6.8 \pm 2.3$ & 10 & 9 \\
\hline Chen et al. [7] & 2020 & $\mathrm{R}$ & 222 & 88 & $25.0 \pm 7.8$ & $4.2 \pm 0.8$ & 14 & 11 \\
\hline Panzram et al. [33] & 2020 & $\mathrm{R}$ & 100 & 76 & $27.8 \pm 1.2$ & $8.3 \pm 2.2$ & 22 & 8 \\
\hline Yian et al. [58] & 2020 & $\mathrm{R}$ & 337 & 83 & - & $6.2 \pm 1.0$ & 30 & 10 \\
\hline Dekker et al. [10] & 2020 & $\mathrm{P}$ & 405 & 89 & $27.5 \pm 4.8$ & $5.1 \pm 0.7$ & 15 & 10 \\
\hline
\end{tabular}

$R$ retrospective, $P$ prospective

difference in recurrence risk when comparing patient with and without hyperlaxity $\left(\mathrm{RR}=1.21\right.$; n.s.; $I^{2}=47 \%$; supplementary 4, Fig. 5).

\section{Sports participation}

Meta-analyses were feasible for participation in competitive, contact and overhead sports. Six studies (1,206 shoulders) demonstrated a higher recurrence risk in patients participating in competitive sports compared to recreational or no sports $\left(\mathrm{RR}=2.47 ; P=0.02 ; I^{2}=72 \%\right.$; Fig. 3$) ; 11$ studies (1,746 shoulders) demonstrated no difference when comparing participation in contact or overhead sports with other or no sports $\left(\mathrm{RR}=1.51\right.$; n.s.; $I^{2}=71 \%$; supplementary 4 , Fig. 6); seven studies (869 shoulders) demonstrated no difference when comparing participation in contact sports only with other or no sports $\left(\mathrm{RR}=1.50\right.$; n.s.; $I^{2}=53 \%$; supplementary 4, Figure 7); five studies (724 shoulders) demonstrated no difference when comparing participation in overhead sports only with other or no sports $(\mathrm{RR}=0.64$; n.s.; $I^{2}=44 \%$; supplementary 4 , Figure 8 ).

\section{Bony lesions or bone loss}

Meta-analyses were feasible for presence of a Hill-Sachs lesion or glenoid bone loss, glenoid fracture and an offtrack Hill-Sachs lesion. Fourteen studies (2113 shoulders) demonstrated a higher recurrence risk in patients with a Hill-Sachs lesion compared to patients where the lesions was not reported $\left(\mathrm{RR}=1.74 ; P=0.0005 ; I^{2}=31 \%\right.$; Fig. 4$)$; three studies (667 shoulders demonstrated a higher recurrence risk in patients with an off-track Hill-Sachs lesion compared to patients with an on-track lesion $(\mathrm{RR}=3.24$; $P=0.002 ; I^{2}=84 \%$; supplementary 4, Figure 9); 13 studies 


\begin{tabular}{|c|c|c|c|c|c|c|c|c|c|c|}
\hline Study or Subgroup & \multicolumn{2}{|c|}{ Age $\leq 20$} & \multicolumn{2}{|c|}{ Age $>20$} & \multicolumn{3}{|c|}{ Risk Ratio } & \multirow{2}{*}{\multicolumn{3}{|c|}{$\begin{array}{c}\text { Risk Ratio } \\
\text { M-H, Random, } 95 \% \text { Cl }\end{array}$}} \\
\hline & & & & 128 & & & 2010 & & & \\
\hline rIIIKKIIA Et al ZU IO & 10 & 20 & 18 & 138 & $8.3 \%$ & $3.61[2.03,0.42]$ & 2010 & & & \\
\hline Voos et al 2010 & 3 & 8 & 8 & 65 & $4.2 \%$ & $3.05[1.01,9.20]$ & 2010 & & & \\
\hline Phadnis et al 2015 & 11 & 37 & 8 & 104 & $5.9 \%$ & $3.86[1.69,8.86]$ & 2015 & & & \\
\hline Aboalata et al 2017 & 9 & 23 & 18 & 120 & $7.4 \%$ & $2.61[1.34,5.07]$ & 2017 & & & \\
\hline Nakagawa et al 2017 & 22 & 82 & 1 & 31 & $1.7 \%$ & $8.32[1.17,59.10]$ & 2017 & & & \\
\hline Lee et al 2018 & 20 & 116 & 12 & 54 & $7.7 \%$ & $0.78[0.41,1.47]$ & 2018 & & & \\
\hline Loppini et al 2019 & 31 & 116 & 83 & 554 & $10.7 \%$ & $1.78[1.24,2.56]$ & 2019 & & & \\
\hline Ono et al 2019 & 7 & 14 & 9 & 37 & $6.4 \%$ & $2.06[0.95,4.45]$ & 2019 & & & \\
\hline Vermeulen et al 2019 & 16 & 31 & 17 & 116 & $8.5 \%$ & $3.52[2.02,6.14]$ & 2019 & & & \\
\hline Iban et al 2019 & 0 & 13 & 20 & 129 & $0.9 \%$ & $0.23[0.01,3.55]$ & 2019 & & & \\
\hline Thomazeau et al 2019 & 8 & 19 & 16 & 106 & $7.1 \%$ & $2.79[1.39,5.58]$ & 2019 & & & \\
\hline Panzram et al 2020 & 8 & 29 & 14 & 71 & $6.6 \%$ & $1.40[0.66,2.97]$ & 2020 & & & \\
\hline Yian et al 2020 & 55 & 153 & 47 & 184 & $11.1 \%$ & $1.41[1.02,1.95]$ & 2020 & & & 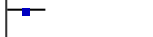 \\
\hline Total $(95 \% \mathrm{Cl})$ & & 742 & & 1997 & $100.0 \%$ & $2.02[1.54,2.65]$ & & & & \\
\hline Total events & 219 & & 322 & & & & & & & \\
\hline \multicolumn{8}{|c|}{$\begin{array}{l}\text { Heterogeneity: } \mathrm{Tau}^{2}=0.15 ; \mathrm{Chi}^{2}=33.56, \mathrm{df}=14(P=0.002) ; \mathrm{I}^{2}=58 \% \\
\text { Test for overall effect: } Z=5.07(P<0.00001)\end{array}$} & 0.01 & $\begin{array}{l}0.1 \\
\text { Favours } \leq 20\end{array}$ & $\begin{array}{l}10 \\
\text { Favours }>20\end{array}$ \\
\hline
\end{tabular}

Fig. 2 Meta-analysis of risk factor age $\leq 20$ years

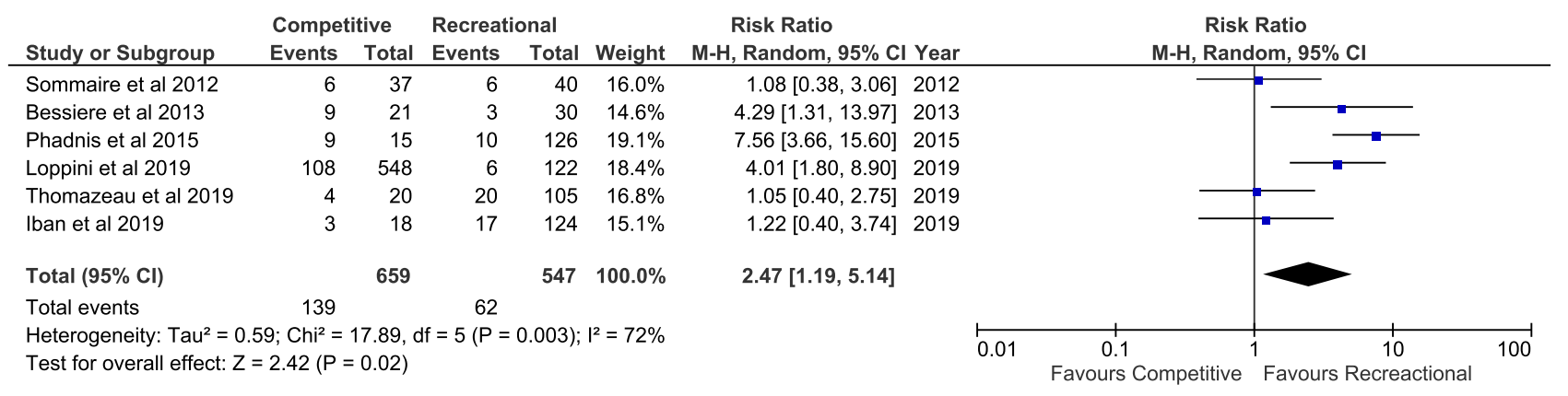

Fig. 3 Meta-analysis of risk factor participation in competitive sports

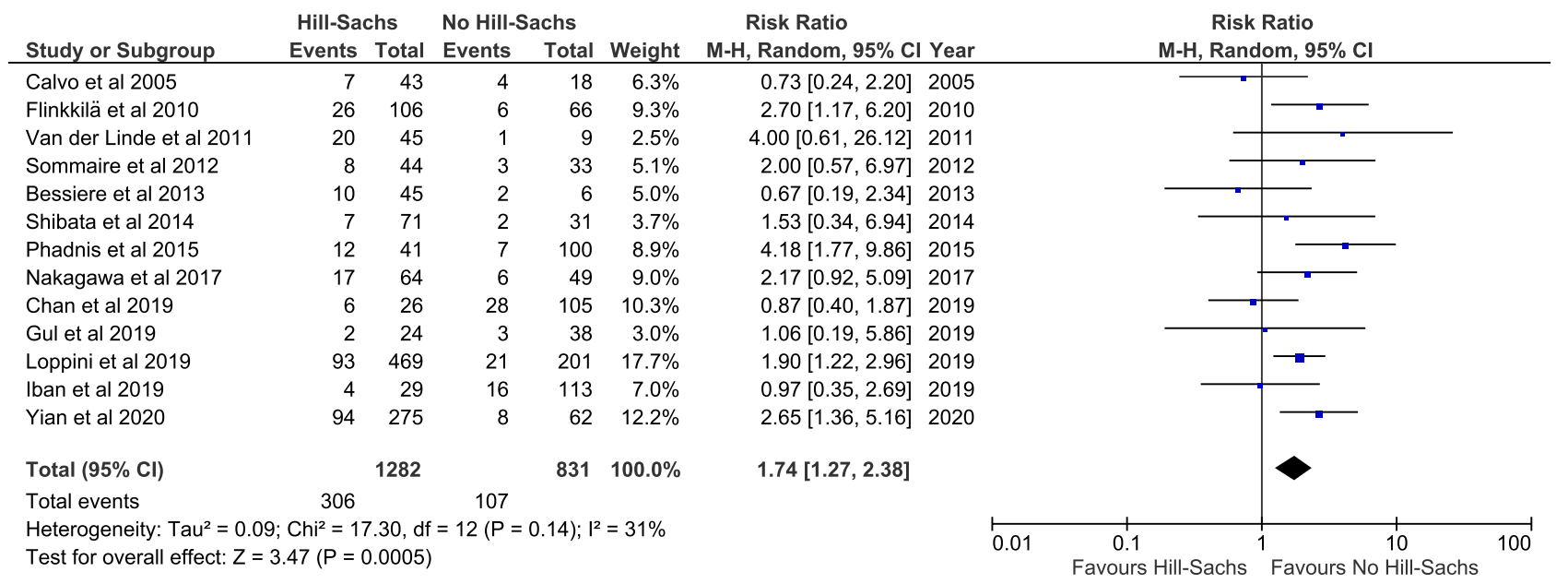

Fig. 4 Meta-analysis of risk factor Hill-Sachs lesion 


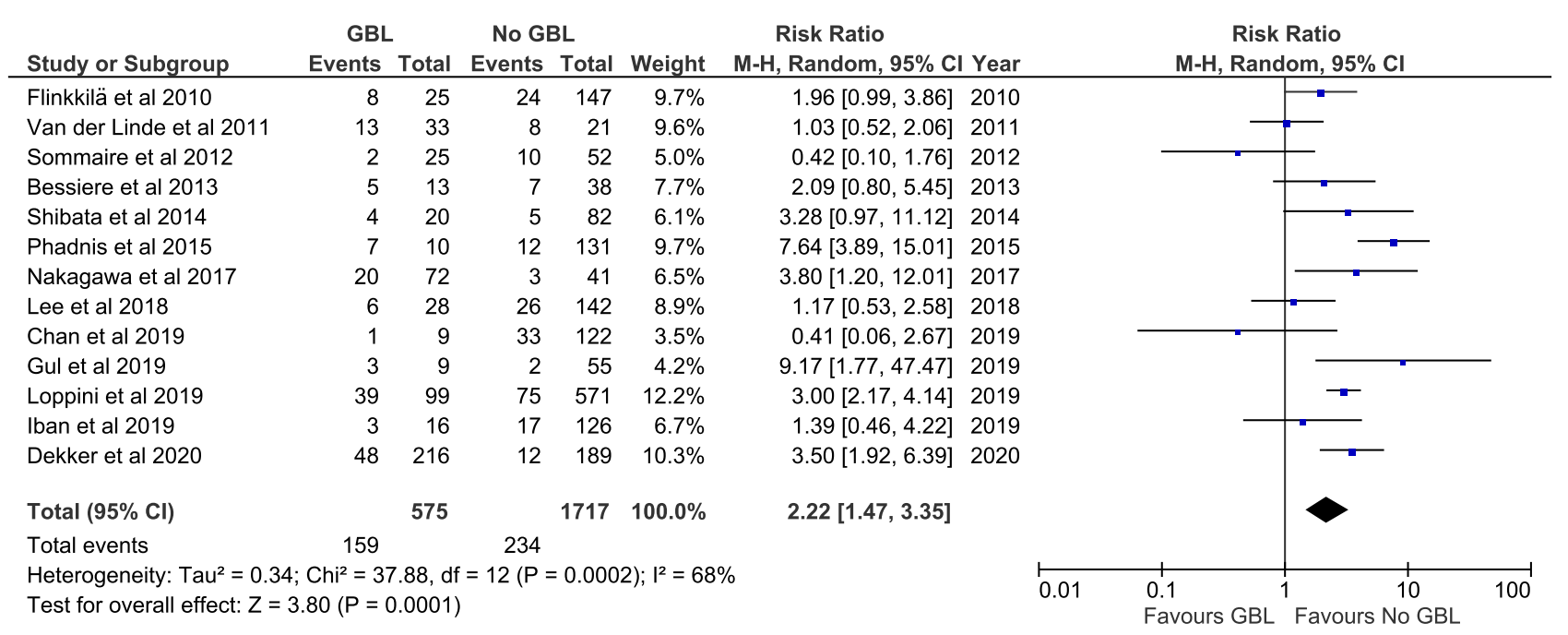

Fig. 5 Meta-analysis of risk factor glenoid bone loss. GBL= glenoid bone loss

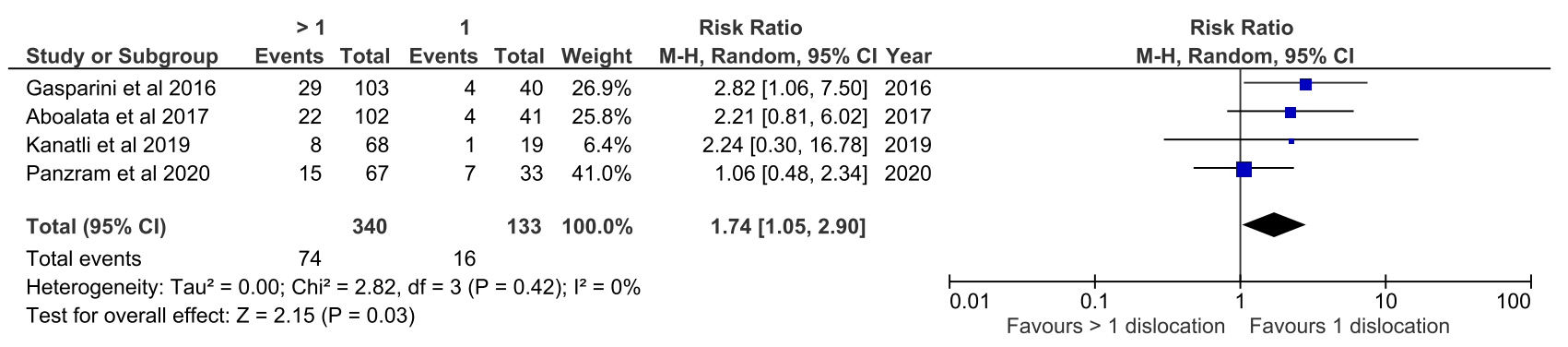

Fig. 6 Meta-analysis of risk factor $>1$ preoperative dislocations

(2113 shoulders) demonstrated a higher recurrence risk in patients with a glenoid bone loss compared to patients without bone loss (RR = 2.22; $P=0.0001 ; I^{2}=68 \%$; Fig. 5); four studies (338 shoulders) demonstrated no difference when comparing patients with a glenoid fracture to patients where the fracture was not reported (RR $=1.01$; n.s.; $I^{2}=34 \%$; supplementary 4, Figure 10).

\section{Soft-tissue lesions}

Meta-analyses were feasible for presence of an ALPSA, any SLAP lesion, SLAP lesion with repair and a rotator cuff lesion. Three studies (523 shoulders) demonstrated a higher recurrence risk in patients with an ALPSA lesion compared to patients where the lesions was not reported $(\mathrm{RR}=1.90$; $P=0.03 ; I^{2}=0 \%$; supplementary 4, Figure 11$)$; five studies (610 shoulders) demonstrated no difference when comparing patients with any SLAP lesion compared to patients where the lesions was not reported $\left(\mathrm{RR}=0.72 ;\right.$ n.s.; $I^{2}=0 \%$; supplementary 4, Figure 12); three studies (278 shoulders) demonstrated no difference when comparing patients with a SLAP lesion with repair compared to patients where the lesions was not reported and a repair was not indicated $\left(\mathrm{RR}=0.58\right.$; n.s.; $I^{2}=0 \%$; supplementary 4, Figure 13$)$; two studies (344 shoulders) demonstrated no difference when comparing patients with a rotator cuff tear compared to patients where the tear was not reported $(\mathrm{RR}=0.96$; n.s.; $I^{2}=0 \%$; supplementary 4 , Figure 14). A meta-analysis for GLAD lesions was not feasible, however Pogorzelski et al. found a higher recurrence rate in patients with the lesion $(43 \%)$ compared to patients where the lesions was not reported $(13 \%)[36]$.

\section{Number of preoperative dislocations}

Meta-analyses were feasible for $>1$ preoperative dislocations and $>5$ preoperative dislocations. Four studies (473 shoulders) demonstrated a higher recurrence risk in patients with $>1$ dislocations lesion compared to patients with one 
dislocation ( $\mathrm{RR}=1.74 ; P=0.03 ; I^{2}=0 \%$; Fig. 6 ); six studies (567 shoulders) demonstrated no difference when comparing patients with $>5$ dislocations compared to patients with $\leq 5$ dislocations ( $\mathrm{RR}=1.07$; n.s.; $I^{2}=59 \%$; supplementary 4 , Figure 15).

\section{Time from first-time dislocation to surgical treatment and number of anchors}

Meta-analyses were feasible for surgical delay of $>6$ months from first-time dislocation to surgery and using $\leq 2$ anchors during surgery. Two studies (565 shoulders) demonstrated a higher recurrence risk in patients with $>6$ months delay compared to patients that received surgery within 6 months $\left(\mathrm{RR}=2.86 ; P<0.0001 ; I^{2}=0 \%\right.$; supplementary 4 , Figure 16); four studies (526 shoulders) demonstrated no difference when comparing patients with $\leq 2$ anchors to patients with $>2$ anchors $\left(\mathrm{RR}=1.57\right.$; n.s.; $I^{2}=25 \%$; supplementary 4, Figure 17).

\section{ISIS and MSTS}

Meta-analyses were feasible for both ISIS $>3$ and ISIS $>6$. Seven studies (1,380 shoulders demonstrated a higher recurrence risk in patients with an ISIS $>3$ compared to patients with an ISIS $\leq 3\left(\mathrm{RR}=3.28 ; P=0.0007 ; I^{2}=77 \%\right.$; supplementary 4, Figure 18); four studies (1136 shoulders) demonstrated a higher recurrence risk in patients with an ISIS $>6$ compared to patients with an ISIS $\leq 6(\mathrm{RR}=4.88$; $P<0.00001 ; I^{2}=71 \%$; supplementary 4, Figure 19). A metaanalysis for MSTS was not feasible, however, Kanatli et al. found a higher recurrence rate in patients with the MSTS (31\%) compared to patients where the sign was not reported (7\%)[23].

\section{Discussion}

The most important findings of the present study were that age $\leq 20$ years, age $\leq 30$ years, participation in competitive sports, a Hill-Sachs lesion, an off-track Hill-Sachs lesion, glenoid bone loss, an ALPSA lesion, $>1$ preoperative dislocations, $>6$ months surgical delay from first-time dislocation to surgery, ISIS $>3$ and ISIS $>6$ were risk factors for recurrence following an arthroscopic Bankart repair. An off-track Hill-Sachs lesion and ISIS $>3$ demonstrated the highest risk of recurrence with a RR $>3$. Male gender, an affected dominant arm, hyperlaxity, participation in contact and/or overhead sports, a glenoid fracture, a SLAP lesion, a rotator cuff tear, $>5$ preoperative dislocations and using $\leq 2$ anchors could not be confirmed as risk factors for recurrence. In addition, no difference was observed between the age groups $\leq 20$ years and $21-30$ years.
This systematic review and meta-analysis includes several limitations. First, an $\mathrm{I}^{2}$ statistic of $>50 \%$ was observed in 11 meta-analyses [18]. This can be due to differences in study design, definitions or patient selection. For example, definition of hyperlaxity was not identical amongst studies, as different hyperlaxity tests were used. Second, this meta-analysis pooled averages and could not use individual patient data. This explains why the risk factors age $\leq 20$ and $\leq 30$ could only be analyzed separately and a multivariate analysis was not feasible. Third, most included studies had a retrospective design, inherently associated with missing data or biased outcomes. Fourth, this systematic review pooled the data of each risk factor, however the severity could not be included. Lesions vary in size and morphology, influencing recurrence risk [10]. Due to these limitations, the results should be interpreted with caution. However, this review includes strengths as well. A systematic approach was used and the search was constructed in cooperation with an information specialist. In addition, this is the first review that quantitatively evaluates these factors.

This review included the glenoid track concept and ISIS, which have both been validated in previous studies [2, 40, $41,56]$. The glenoid track concept takes both glenoid and humeral bone loss into account and may demonstrate when a bone augmentation procedure should be performed, as offtrack lesions were confirmed as an important risk factor for recurrence following a Bankart repair. However, this cut-off value does not seem suitable to determine if a soft tissue repair is more beneficial than non-operative treatment for patients with small to no bone defects. A higher ISIS can be used to estimate recurrence risk, but the tool is probably too simplistic to determine a cut-off value that demonstrates when operative treatment is beneficial $[6,22,35]$. In addition, both type of sport and hyperlaxity are included in the ISIS and could not be confirmed as risk factors for recurrence in the quantitative analyses. Therefore, these items may need to be substituted by other factors or adjusted to be of additional value in this tool. For example, Nakagawa et al. demonstrated that the recurrence rate is significantly higher in rugby players compared to other collision or contact sports in competitive athletes [30]. This may indicate that contact and overhead sports should not be pooled and a more differentiated approach is needed for type of sport to be used in the ISIS. Moreover, SLAP lesions with/without repair and rotator cuff lesions were included in the analyses and could not be confirmed as risk factors. This in line with the recent meta-analysis by Feng et al. demonstrating that a combined SLAP and Bankart repair does not decrease recurrence rates compared to a Bankart repair alone [13]. The current metaanalysis for rotator cuff tears only included 19 cases and this sample size might have been too small to observe a difference. The incidence of rotator cuff lesions increases 
with age and it has been shown that older age is associated with lower recurrence rates [39, 50]. It is unclear if young patients with a rotator cuff tear demonstrate a higher risk for recurrence. As an accurate tool that can determine failure following treatment is lacking, the benefits and risks should be discussed with patients to make a shared decision.

Studies that determine risk factors for recurrence generally use dislocation or subluxation as objective failure following treatment or use a combination of objective and subjective failure as outcome [41]. Defining when treatment for anterior shoulder dislocations has failed can be challenging and an international consensus has yet to be reached. Park et al. have demonstrated that some factors, such as width of the Hill-Sachs lesion and number of preoperative dislocations, may increase the risk of patient dissatisfaction without recurrence following arthroscopic stabilization [34]. These factors did not necessarily match the factors that predicted objective failure. A different treatment strategy may have been more suitable for these patients even though they did not experience objective failure. Separately taking objective and subjective failure into account may both reduce social costs and increase patient satisfaction [9, 34, 47].

This meta-analysis pooled study data to identify risk factors and allow for more consensus regarding these factors. This can help professionals decide if a more invasive procedure (e.g. bone augmentation) is more beneficial for a patient to prevent recurrence compared to a labral repair. Future research should focus on clear definitions for risk factors and patient selection, preferably on an international scale. Prospective cohort study designs with a large sample size should be used to confirm if the factors identified in this review predispose recurrence following an arthroscopic Bankart repair. These studies should separately include subjective failure as well as objective failure to increase patient satisfaction and reduce social costs [9, $34,47]$. Currently, it is uncommon to publish anonymous individual patient data in orthopedic research. Sharing this data can be of additional value for pooling results in metaanalyses to increase samples sizes and homogeneity of the analyses [12]. In addition, this data can be used to create models that can determine recurrence risk for individual patients based on their profile [26].

\section{Conclusion}

Meta-analyses demonstrated that age $\leq 20$ years, age $\leq 30$ years, participation in competitive sports, HillSachs lesion, off-track Hill-Sachs lesion, glenoid bone loss, ALPSA lesion, $>1$ preoperative dislocations, $>6$ months surgical delay from first-time dislocation to surgery, ISIS $>3$ and ISIS $>6$ were risk factors for recurrence following an arthroscopic Bankart repair. These factors can assist clinicians in giving a proper advice regarding treatment.

Supplementary Information The online version contains supplementary material available at https://doi.org/10.1007/s00167-021-06704-7.

Acknowledgements The authors would like to thank Dr. Nourissat and his research group for sharing the data of the French Society of Arthroscopy (SFA) and Rennes University Hospital (CHU).

Author contributions LPEV: Lead author, design, search, data acquisition, data-analysis, data interpretation, writing. SHS: Design, search, data acquisition, writing. AG: Design, search, data acquisition. GMMJK: Design, data-interpretation, writing. SP-VK: Design, search. DFPD: Design, data-interpretation, writing. MPJB: Design, data-interpretation, writing.

Funding The lead author (Lukas P.E. Verweij) received a grant from the Amsterdam UMC to complete his PhD. This is a personal grant and is not related to commercial entities (https://www.amc.nl/web/leren/ graduate-school/phd-1/mdphd-scholarship-amc.htm).

\section{Declarations}

Conflict of interest The authors declare no conflict of interest.

Ethical approval Not available.

Informed consent Not available.

Open Access This article is licensed under a Creative Commons Attribution 4.0 International License, which permits use, sharing, adaptation, distribution and reproduction in any medium or format, as long as you give appropriate credit to the original author(s) and the source, provide a link to the Creative Commons licence, and indicate if changes were made. The images or other third party material in this article are included in the article's Creative Commons licence, unless indicated otherwise in a credit line to the material. If material is not included in the article's Creative Commons licence and your intended use is not permitted by statutory regulation or exceeds the permitted use, you will need to obtain permission directly from the copyright holder. To view a copy of this licence, visit http://creativecommons.org/licenses/by/4.0/.

\section{References}

1. Aboalata M, Plath JE, Seppel G, Juretzko J, Vogt S, Imhoff AB (2017) Results of arthroscopic Bankart repair for anterior-inferior shoulder instability at 13-year follow-up. Am J Sports Med 45:782-787

2. Balg F, Boileau P (2007) The instability severity index score. A simple pre-operative score to select patients for arthroscopic or open shoulder stabilisation. J Bone Joint Surg Br 89:1470-1477

3. Bessiere C, Trojani C, Pelegri C, Carles M, Boileau P (2013) Coracoid bone block versus arthroscopic Bankart repair: a comparative paired study with 5-year follow-up. Orthop Traumatol Surg Res 99:123-130

4. Bouliane M, Saliken D, Beaupre LA, Silveira A, Saraswat MK, Sheps DM (2014) Evaluation of the Instability Severity Index Score and the Western Ontario Shoulder Instability Index as 
predictors of failure following arthroscopic Bankart repair. Bone Joint J 96-B:1688-1692

5. Calvo E, Granizo JJ, Fernandez-Yruegas D (2005) Criteria for arthroscopic treatment of anterior instability of the shoulder: a prospective study. J Bone Joint Surg Br 87:677-683

6. Chan AG, Kilcoyne KG, Chan S, Dickens JF, Waterman BR (2019) Evaluation of the instability severity index score in predicting failure following arthroscopic Bankart surgery in an active military population. J Shoulder Elbow Surg 28:e156-e163

7. Chen KH, Yang TC, Chiang ER, Wang HY, Ma HL (2021) The Hill-Sachs interval to glenoid track width ratio is comparable to the instability severity index score for predicting risk of recurrent instability after arthroscopic Bankart repair. Knee Surg Sports Traumatol Arthrosc 29:250-256

8. Cools AM, Borms D, Castelein B, Vanderstukken F, Johansson FR (2016) Evidence-based rehabilitation of athletes with glenohumeral instability. Knee Surg Sports Traumatol Arthrosc 24:382-389

9. Crall TS, Bishop JA, Guttman D, Kocher M, Bozic K, Lubowitz JH (2012) Cost-effectiveness analysis of primary arthroscopic stabilization versus nonoperative treatment for first-time anterior glenohumeral dislocations. Arthroscopy 28:1755-1765

10. Dekker TJ, Peebles LA, Bernhardson AS, Rosenberg SI, Murphy CP, Golijanin P et al (2020) Risk factors for recurrence after arthroscopic instability repair-the importance of glenoid bone loss $>15 \%$, patient age, and duration of symptoms: a matched cohort analysis. Am J Sports Med 48:3036-3041

11. Di Giacomo G, Itoi E, Burkhart SS (2014) Evolving concept of bipolar bone loss and the Hill-Sachs lesion: from "engaging/ non-engaging" lesion to "on-track/off-track" lesion. Arthroscopy 30:90-98

12. El Emam K, Rodgers S, Malin B (2015) Anonymising and sharing individual patient data. BMJ 350:h1139

13. Feng S, Song Y, Li H, Chen J, Chen J, Chen S (2019) Outcomes for arthroscopic repair of combined Bankart/SLAP lesions in the treatment of anterior shoulder instability: a systematic review and meta-analysis. Orthop J Sports Med 7:2325967119877804

14. Flinkkila T, Hyvonen P, Ohtonen P, Leppilahti J (2010) Arthroscopic Bankart repair: results and risk factors of recurrence of instability. Knee Surg Sports Traumatol Arthrosc 18:1752-1758

15. Gasparini G, De Benedetto M, Cundari A, De Gori M, Orlando N, McFarland EG et al (2016) Predictors of functional outcomes and recurrent shoulder instability after arthroscopic anterior stabilization. Knee Surg Sports Traumatol Arthrosc 24:406-413

16. Gul O, Okutan AE, Ayas MS (2019) Arthroscopic glenoid labral lesion repair using all-suture anchor for traumatic anterior shoulder instability: short-term results. J Shoulder Elbow Surg 28:1991-1997

17. Hayashida K, Yoneda M, Nakagawa S, Okamura K, Fukushima S (1998) Arthroscopic Bankart suture repair for traumatic anterior shoulder instability: analysis of the causes of a recurrence. Arthroscopy 14:295-301

18. Higgins JP, Thompson SG (2002) Quantifying heterogeneity in a meta-analysis. Stat Med 21:1539-1558

19. Hovelius L, Olofsson A, Sandstrom B, Augustini BG, Krantz L, Fredin $\mathrm{H}$ et al (2008) Nonoperative treatment of primary anterior shoulder dislocation in patients forty years of age and younger. A prospective twenty-five-year follow-up. J Bone Joint Surg Am 90:945-952

20. Hozo SP, Djulbegovic B, Hozo I (2005) Estimating the mean and variance from the median, range, and the size of a sample. BMC Med Res Methodol 5:13

21. Hurley ET, Lim Fat D, Farrington SK, Mullett H (2019) Open versus arthroscopic latarjet procedure for anterior shoulder instability: a systematic review and meta-analysis. Am J Sports Med 47:1248-1253
22. Iban MAR, Gismero CVA, Marco SM, Diaz RR, Hernndez TD, Bello GD et al (2019) Instability severity index score values below 7 do not predict recurrence after arthroscopic Bankart repair. Knee Surg Sports Traumatol Arthrosc 27:3905-3911

23. Kanatli U, Ozer M, Gem M, Ozturk BY, Ataoglu MB, Cetinkaya $\mathrm{M}$ et al (2019) Multiple subscapularis tendon sign: a new risk factor for recurrence after arthroscopic anterior shoulder instability surgery. Orthop J Sports Med 7:2325967119853507

24. Lee SH, Lim KH, Kim JW (2018) Risk factors for recurrence of anterior-inferior instability of the shoulder after arthroscopic bankart repair in patients younger than 30 years. Arthroscopy 34:2530-2536

25. Loppini M, Delle Rose G, Borroni M, Morenghi E, Pitino D, Zamora CD et al (2019) Is the instability severity index score a valid tool for predicting failure after primary arthroscopic stabilization for anterior glenohumeral instability? Arthroscopy 35:361-366

26. Machine Learning Consortium obotS F Investigators (2021) A machine learning algorithm to identify patients with tibial shaft fractures at risk for infection after operative treatment. J Bone Joint Surg Am 103:532-540

27. Matsumura N, Oki S, Fukasawa N, Matsumoto M, Nakamura M, Nagura $\mathrm{T}$ et al (2019) Glenohumeral translation during active external rotation with the shoulder abducted in cases with glenohumeral instability: a 4-dimensional computed tomography analysis. J Shoulder Elbow Surg 28:1903-1910

28. Moher D, Liberati A, Tetzlaff J, Altman DG, Group P (2009) Preferred reporting items for systematic reviews and meta-analyses: the PRISMA statement. PLoS Med 6:e1000097

29. Moroder P, Damm P, Wierer G, Bohm E, Minkus M, Plachel F et al (2019) Challenging the current concept of critical glenoid bone loss in shoulder instability: does the size measurement really tell it all? Am J Sports Med 47:688-694

30. Nakagawa S, Mae T, Sato S, Okimura S, Kuroda M (2017) Risk factors for the postoperative recurrence of instability after arthroscopic Bankart repair in athletes. Orthop J Sports Med 5:2325967117726494

31. Ono Y, Davalos Herrera DA, Woodmass JM, Lemmex DB, Carroll MJ, Yamashita S et al (2019) Long-term outcomes following isolated arthroscopic Bankart repair: a 9- to 12-year follow-up. JSES Open Access 3:189-193

32. Ouzzani M, Hammady H, Fedorowicz Z, Elmagarmid A (2016) Rayyan-a web and mobile app for systematic reviews. Syst Rev 5:210

33. Panzram B, Kentar Y, Maier M, Bruckner T, Hetto P, Zeifang F (2020) Mid-term to long-term results of primary arthroscopic Bankart repair for traumatic anterior shoulder instability: a retrospective study. BMC Musculoskelet Disord 21:191

34. Park I, Kang JS, Jo YG, Shin SJ (2019) Factors related to patient dissatisfaction versus objective failure after arthroscopic shoulder stabilization for instability. J Bone Joint Surg Am 101:1070-1076

35. Phadnis J, Arnold C, Elmorsy A, Flannery M (2015) Utility of the instability severity index score in predicting failure after arthroscopic anterior stabilization of the shoulder. Am J Sports Med 43:1983-1988

36. Pogorzelski J, Fritz EM, Horan MP, Katthagen JC, Provencher MT, Millett PJ (2018) Failure following arthroscopic Bankart repair for traumatic anteroinferior instability of the shoulder: is a glenoid labral articular disruption (GLAD) lesion a risk factor for recurrent instability? J Shoulder Elbow Surg 27:e235-e242

37. Porcellini G, Campi F, Pegreffi F, Castagna A, Paladini P (2009) Predisposing factors for recurrent shoulder dislocation after arthroscopic treatment. J Bone Joint Surg Am 91:2537-2542

38. Randelli P, Ragone V, Carminati S, Cabitza P (2012) Risk factors for recurrence after Bankart repair a systematic review. Knee Surg Sports Traumatol Arthrosc 20:2129-2138 
39. Robinson CM, Shur N, Sharpe T, Ray A, Murray IR (2012) Injuries associated with traumatic anterior glenohumeral dislocations. J Bone Joint Surg Am 94:18-26

40. Rouleau DM, Hebert-Davies J, Djahangiri A, Godbout V, Pelet S, Balg F (2013) Validation of the instability shoulder index score in a multicenter reliability study in 114 consecutive cases. Am J Sports Med 41:278-282

41. Shaha JS, Cook JB, Rowles DJ, Bottoni CR, Shaha SH, Tokish JM (2016) Clinical validation of the glenoid track concept in anterior glenohumeral instability. J Bone Joint Surg Am 98:1918-1923

42. Shaha JS, Cook JB, Song DJ, Rowles DJ, Bottoni CR, Shaha SH et al (2015) Redefining "Critical" bone loss in shoulder instability: functional outcomes worsen with "Subcritical" bone loss. Am J Sports Med 43:1719-1725

43. Shibata H, Gotoh M, Mitsui Y, Kai Y, Nakamura H, Kanazawa T et al (2014) Risk factors for shoulder re-dislocation after arthroscopic Bankart repair. J Orthop Surg Res 9:53

44. Slim K, Nini E, Forestier D, Kwiatkowski F, Panis Y, Chipponi J (2003) Methodological index for non-randomized studies (MINORS): Development and validation of a new instrument. ANZ J Surg 73:712-716

45. Sommaire C, Penz C, Clavert P, Klouche S, Hardy P, Kempf JF (2012) Recurrence after arthroscopic Bankart repair: Is quantitative radiological analysis of bone loss of any predictive value? Orthop Traumatol Surg Res 98:514-519

46. Thomazeau H, Langlais T, Hardy A, Curado J, Herisson O, Mouton J et al (2019) Long-term, prospective, multicenter study of isolated bankart repair for a patient selection method based on the instability severity index score. Am J Sports Med 47:1057-1061

47. van der Linde JA, Bosmans JE, Ter Meulen DP, van Kampen DA, van Deurzen DF, Haverlag R et al (2019) Direct and indirect costs associated with nonoperative treatment for shoulder instability: an observational study in 132 patients. Shoulder Elbow 11:265-274

48. van der Linde JA, van Kampen DA, Terwee CB, Dijksman LM, KleinJan G, Willems WJ (2011) Long-term results after arthroscopic shoulder stabilization using suture anchors an 8-to 10-year follow-up. Am J Sports Med 39:2396-2403

49. Vermeulen AE, Landman EBM, Veen EJD, Nienhuis S, Koorevaar CT (2019) Long-term clinical outcome of arthroscopic Bankart repair with suture anchors. J Shoulder Elbow Surg 28:E137-E143

50. Verweij LP, Baden DN, van der Zande JM, van den Bekerom MP (2020) Assessment and management of shoulder dislocation. BMJ 371:m4485

51. Verweij LPE, Pruijssen EC, Kerkhoffs G, Blankevoort L, Sierevelt IN, van Deurzen DFP et al (2020) Treatment type may influence degree of post-dislocation shoulder osteoarthritis: a systematic review and meta-analysis. Knee Surg Sports Traumatol Arthrosc. https://doi.org/10.1007/s00167-020-06263-3

52. Verweij LPE, Schuit AA, Kerkhoffs G, Blankevoort L, van den Bekerom MPJ, van Deurzen DFP (2020) Accuracy of currently available methods in quantifying anterior glenoid bone loss: controversy regarding gold standard-a systematic review. Arthroscopy 36:2295-2313 e2291

53. Voos JE, Livermore RW, Feeley BT, Altchek DW, Williams RJ, Warren RF et al (2010) Prospective evaluation of arthroscopic bankart repairs for anterior instability. Am J Sports Med 38:302-307

54. Walter SD, Yao X (2007) Effect sizes can be calculated for studies reporting ranges for outcome variables in systematic reviews. J Clin Epidemiol 60:849-852

55. Williams HLM, Evans JP, Furness ND, Smith CD (2019) It's not all about redislocation: a systematic review of complications after anterior shoulder stabilization surgery. Am J Sports Med 47:3277-3283

56. Yamamoto N, Itoi E, Abe H, Minagawa H, Seki N, Shimada Y et al (2007) Contact between the glenoid and the humeral head in abduction, external rotation, and horizontal extension: A new concept of glenoid track. J Shoulder Elbow Surg 16:649-656

57. Yang TC, Chen KH, Chiang ER, Chang MC, Ma HL (2018) Using the "Hill-Sachs interval to glenoid track width ratio" for prediction of recurrent instability after arthroscopic Bankart repair. Orthop Traumatol Surg Res 104:797-801

58. Yian EH, Weathers M, Knott JR, Sodl JF, Spencer HT (2020) Predicting failure after primary arthroscopic bankart repair: analysis of a statistical model using anatomic risk factors. Arthroscopy 36:964-970

59. Zacchilli MA, Owens BD (2010) Epidemiology of shoulder dislocations presenting to emergency departments in the United States. J Bone Joint Surg Am 92:542-549

60. Zaremski JL, Galloza J, Sepulveda F, Vasilopoulos T, Micheo W, Herman DC (2017) Recurrence and return to play after shoulder instability events in young and adolescent athletes: a systematic review and meta-analysis. Br J Sports Med 51:177-184

Publisher's Note Springer Nature remains neutral with regard to jurisdictional claims in published maps and institutional affiliations.

\section{Authors and Affiliations}

\section{Lukas P. E. Verweij ${ }^{1,2,3}$. Sanne H. van Spanning ${ }^{4}$. Adriano Grillo ${ }^{4}$ Gino M. M. J. Kerkhoffs ${ }^{1,2,3}$. Simone Priester-Vink ${ }^{5}$. Derek F. P. van Deurzen ${ }^{4}$. Michel P. J. van den Bekerom ${ }^{4,6}$}

1 Amsterdam UMC, Department of Orthopedic Surgery, University of Amsterdam, Amsterdam Movement Sciences, Location AMC, Meibergdreef 9, 1105 AZ Amsterdam, The Netherlands

2 Academic Center for Evidence-Based Sports Medicine (ACES), Amsterdam, The Netherlands

3 Amsterdam Collaboration on Health and Safety in Sports (ACHSS), AMC/VUmc IOC Research Center, Amsterdam, Netherlands
4 Department of Orthopedic Surgery, Shoulder and Elbow Unit, OLVG, Amsterdam, The Netherlands

5 Department of Research and Epidemiology, OLVG, Amsterdam, The Netherlands

6 Faculty of Behavioural and Movement Sciences, Department of Human Movement Sciences, Vrije Universiteit Amsterdam, Amsterdam Movement Sciences, Amsterdam, the Netherlands 